\title{
Necessity and feasibility of care training assistant robot (CaTARo) as shoulder complex joint with multi-DOF in elderly care education
}

Miran Lee ${ }^{1}$, Ko Ameyama ${ }^{1}$, Hirotake Yamazoe ${ }^{2}$ and Joo-Ho Lee ${ }^{2^{*}}$ (0)

\begin{abstract}
As the geriatric population expands, caregivers require more accurate training to handle and care for the elderly. However, students lack methods for acquiring the necessary skill and experience, as well as sufficient opportunities to practice on real human beings. To investigate the necessity and feasibility of care training assistant robots in care education, we developed a simulated robot as a shoulder complex joint with multi-DOF. In this study, five experts with years of experience in elderly care participated in the data-acquisition process, to acquire information on aspects such as the glenohumeral angle, as well as the sterno-clavicular joint and its torque. The experts performed three types of range-of-motion exercises: (i) elevation-depression of sterno-clavicular joint; (ii) extension and flexion of glenohumeral joint; (iii) lateral and medial rotation of glenohumeral joint. The experimental results showed that the quantitative results for all the exercises were significantly different between the experts. Moreover, we observed that even experienced professionals need consistent care education based on quantitative data and feedback. Thus, we confirmed the necessity and feasibility of the care training assistant robot for improving the skills required for elderly care.
\end{abstract}

\section{Introduction}

Caregivers or therapists for the elderly are becoming increasingly important with the continued development of medical technology and the expanding geriatric population [1]. Most students who wish to become caregivers or therapists can receive training at school for some time to improve their skills, whereby they can acquire caregiving experience by watching videos, reading books, training on high-fidelity medical mannequins [2-4], and/or through role-playing to simulate caregiving for an elderly patient [5]. However, these methods are not sufficiently effective for improving the care training performance, because a medical mannequin is unable to record quantitative data on movement, such as the joint angle, force, speed, duration, and pressure value; thus, evaluating the

\footnotetext{
${ }^{*}$ Correspondence: leejooho@is.ritsumei.ac.jp

${ }^{2}$ College of Information Science and Engineering, Ritsumeikan University,

Kusatsu-Shi, Shiga-Ken 5258577, Japan

Full list of author information is available at the end of the article
}

efficacy of care education for trainees becomes difficult. The role-playing method is ineffective because a healthy person cannot accurately simulate the actions of a patient suffering from declining muscle strength and paralysis [6]. Typically, the best training technique involves practicing on an actual human subject. However, continuously recruiting elderly subjects for care education is challenging, and the elderly participants may experience fatigue or boredom from repetitive tests. Therefore, the development of a simulated robotic device for elderly care training is necessary.

The passive range of motion ( $\mathrm{ROM}$ ) exercise is one most important care education task for the elderly. The ROM exercise describes physical exercise to improve the joint movements, which is characterized by the action of ligaments, tendons, muscles, and the joint itself when performing the exercise [7]. The elderly may have limited joint movements in daily life due to stiffness, contraction or weakness of muscles, joints, tendons, or ligaments. 
Therefore, caregivers or therapists must periodically ask the elderly patient to exercise, which may include passive ROM exercise or stretching. Unfortunately, it could be very difficult for elderly care students to gain adequate training and experience in performing $\mathrm{ROM}$ exercises with a real subject. Therefore, the development of joint robotic simulator becomes important, which allows trainees and students to practice ROM exercises effectively, as well as receive quantitative feedback on their performance.

Till date, many previous studies developed simulated robot for various medical training procedures. The experimental methods for simulated robot in care and nurse education were generally classified into three groups such as: (i) feasibility testing of the simulated robot (ii) correlation evaluation between the simulated robot and a real human; (iii) performance comparison between the experts and novices (students) when using the simulated robot. Firstly, studies in various medical field have examined the feasibility of simulation robots. Fujisawa et al. [8] proposed an upper limb patient simulator in practical training for feasibility testing. Their simulator reproduced the stiffness of the elbow joint to allow a trainee to identify the opportunities to stretch when performing physical therapy. Mouri et al. [9] developed and tested a robot hand with disability (contracture) for rehabilitation therapist training through distributed tactile sensors that estimates the elbow joint torque of the robot hand. Wang et al. [10] proposed and simulated an arm robot for neurologic examination training to enhance the skills of medical staff. Huang et al. [11] proposed a simulated patient robot to transfer the patient from bed to wheelchair, while Kitgawa et al. [12] presented a system of humanlike patient robot with emotions to facilitate training in administering injections. Takashina et al. [13] and Gordon [14] developed a patient simulator for cardiac diseases. Wang et al. [15] introduced an arm robot for neurologic examination training and assessed the survey responses from doctors, while Takanobu et al. [16] proposed a dental patient robot with questionnaire assessment. Gerling et al. [17] designed a physical simulator for clinical prostate examination training and evaluated the training effectiveness in pre- and post-test. Ren et al. [18] proposed a multi-joint upper limb simulator with spasticity that can diagnose a disease, perform a stretching exercise, and classify the stiffness result for a patient in pre- and post-evaluation, and concluded that the feasibility of the integrated capabilities was demonstrated through experiments with stroke survivors and healthy subjects. Lin et al. [19] investigated the learning transfer effect of practice on practice-similar and practice-dissimilar skills from one patient to another using a robot patient, and confirmed the necessity of simulated training with robots to assist and improve the training skills for students and novices.

Secondly, examining the correlation between a simulated robot and a real human can help to evaluate the applicability of the simulated robot. Kim et al. [20] presented a haptic elbow spasticity simulator and compared the robot and in-person questionnaire results for improving the reliability of clinical assessment. Based on their results, they concluded that their proposed haptic recreation of spasticity has the potential to be used as a training tool for standardizing and enhancing reliability of clinical assessment. Huang et al. [6, 21] proposed a robot patient for nursing skills training during stand-up and sit-down actions on a wheel-chair during patient transfer, in addition to performing relevant experimental tests. Their results revealed that the robot patient could successfully simulate the limb actions of a patient according to the operations performed by the nursing teachers and was suitable for nursing-skill training. However, the experimental results varied depending on whether the patient being assisted by the expert was a robot or a human being. In standing up and sitting down, the maximum difference values in the bending angle of the back of the robot patient between experts were roughly $10^{\circ}$ (expert 1 and expert 4 ) and $15^{\circ}$ (expert 1 and expert 2). Park et al. [22] presented a haptic elbow robotic simulator to improve the accuracy and reliability of clinical assessment; in their study, four experts performed clinical assessments on the elbow spasticity of a human patient and the haptic elbow robot. However, the experimental result demonstrated that the stretching speeds $(\dot{\theta}, \mathrm{deg} / \mathrm{s})$ differed slightly between experts during the spasticity assessment.

More recently, Noh et al. [23] compared the results of experts and students using a patient simulated robot assisted airway management system for physician supporting, and objectively determined the operator effectiveness while providing quantitative performances based on experimental results. However, the experts exhibited a large deviation in the results of the majority of the parameters.

Therefore, the following two issues can be assumed to determine the necessity and feasibility of Care Training Assistant Robot (CaTARo) in the care education:

- Did experts with many years of experience participate in the care education in the same manner and behavior?

- Did experts differ in quantitative performances when performing the same type (exercise) of the care education? 
The purpose of this study is to design a simulated elderly robot for care and nursing in the care education, and investigate its necessity and feasibility. Specifically, experts with many years of experience were participated to experiment with some of the exercises required for the care education. We assumed that experts will perform care exercises in a similar movement and behavior, and their quantitative data will not vary significantly when the care training is performed using the proposed robot.

Earlier researches [7, 24, 25] focused on the development of the elbow joint of elderly, while a real-time monitoring program was developed to obtain quantitative data, such as angle, torque, and pressure value of the robot. Additionally, we have investigated the feasibility of that system for assessment of care training from novices. This study extends the scope of our earlier study by designing the upper limb of the robot, which has additionally developed shoulder complex joint that also contains the elbow joint designed previously; and providing additional quantitative results for investigating the feasibility of CaTARo.

\section{Hardware configuration of the proposed system}

Here, we developed the robotic upper limb with glenohumeral (GH), sterno-clavicular (SC), and elbow joint (EJ) based on six degree of freedom (DOF). As shown in Fig. 1, the robotic joints of $\mathrm{GH}$ and $\mathrm{SC}$ were combined into the shoulder complex robot. The flexion-extension, abduction-adduction, and lateral-medial rotation of the GH joint were reproduced by combining three servomotors, while elevation-depression and the horizontal extension-horizontal flexion of the SC joint were reproduced by combining two servomotors. The flexionextension of the EJ was reproduced by one servomotor. The symptoms were reproduced by the torque control and the angles of six servo motors.

\section{Joint configuration of shoulder complex joint}

The joint configuration of the elderly robot is shown in Fig. 1, which has totally six joints: three parts of glenohumeral (GH-1), (GH-2) and (GH-3); two parts of sterno-Clavicular (SC-1) and (SC-2); as well as the elbow joint (EJ). The robot body size of the elderly person was determined based on Human Body Properties Database (HBPD) [26] in Japan, and was set to the average size of an elderly person older than 65 years. Based on the HBPD, the lengths of the upper arm and the forearm, as well as the circumferences of the upper arm and the forearm, together with the weight of the robotic elbow joint, were set to $288 \mathrm{~mm}, 285 \mathrm{~mm}, 220 \mathrm{~mm}, 240 \mathrm{~mm}$, and $0.35 \mathrm{~kg}$, respectively.

These joints were designed based on the forceless joint, because the elderly robot does not need to move actively during the care training. The robotic $\mathrm{GH}$ and $\mathrm{SC}$ joints were developed using six Dynamixel XM-430-W350$\mathrm{R}$ (Robotis Inc., Seoul, South Korea) servo motors. Two 6-axis force sensors PFS303YA301 (Leptrino Inc., Nagano, Japan) were used to measure the torque acting at the robotic joints. The servo motor of the GH joint was used to control the flexion-extension, abductionadduction, and lateral-medial rotation of the robotic GH joint. In addition, a 2-DOF robotic SC joint was developed, whose elevation-depression and horizontal

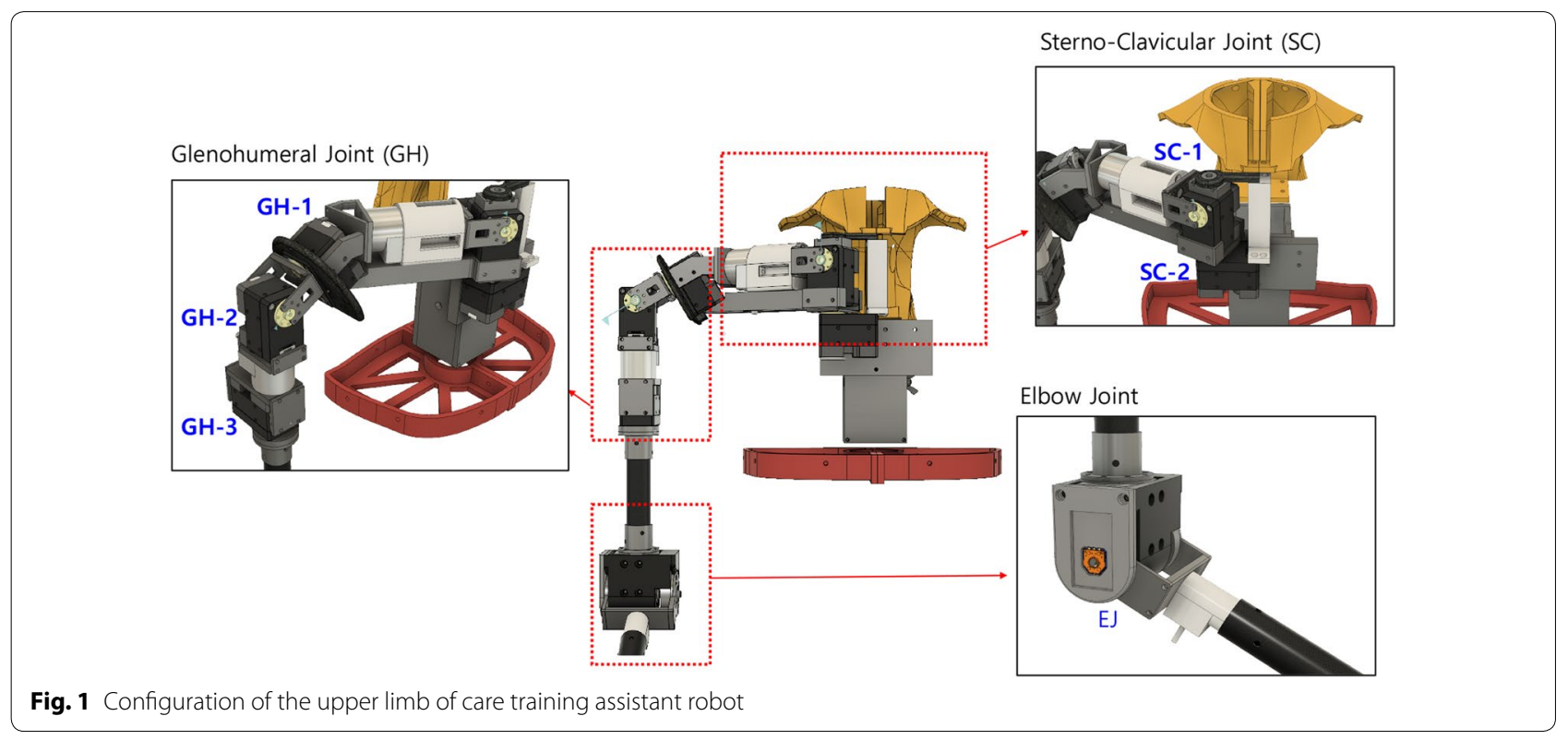


flexion-extension were controlled with the SC joint as the rotation axis.

The robotic EJ was designed based on one servomotor of Dynamixel MX-28 (Robotis Inc., Seoul, South Korea), the 6-axis force sensor of CFS018CA201U (Leptrino Inc., Nagano, Japan), the position angle sensor of SV01L103AEA11T00 (Murata Electronics Co., Ltd., Kyoto, Japan), and four pressure sensors of FlexiForce A201 (Tekscan, Inc., MA, United States). In earlier studies [7, 24, 25], a robotic EJ joint was developed based on 1 -DOF by controlling the flexion and extension of the arm.

The exterior was designed using 3D CAD (Autodesk, Fusion $360^{\circledR}$ ) and created with 3D printer (Zortrax S.A Inc., Lubelska, Poland), whose material was applied based on acrylonitrile butadiene styrene copolymer for robust durability, as shown in Fig. 2a, while the robot surface was covered with a gel (hardness 0 and thickness $10 \mathrm{~mm}$ ) of human skin (Exseal Co., Ltd., Gifu, Japan) to reproduce human-like tactile.

\section{Mechanism of shoulder complex joint}

We focused on three movements, such as elevation and depression (ED) of SC joint, extension-flexion (EF) of GH joint, and lateral-medial rotation (LM) of GH joint, as shown in Fig. 2b. The upper limb robot simulated three movements based on the G1, G2, and SC1 joints. The ED was the upward and downward movements of the shoulders based on the SC1 joints (Fig. 2b, left), and the EF movement was the arm movement of upward and downward using the GH1 joints (Fig. 2b, center), while the LM motion was to rotate the arm outward and inward by the GH2 joint (Fig. 2b, right).

To mimic the human joints, Yamazaki et al. [27] proposed the Eq. (1) of resistance torque by fitting four parameters as $p 1, p 2, p 3$, and $p 4$.

$$
T(\theta)=e^{\left(p_{1}\left(\theta-p_{2}\right)\right)}-e^{\left(p_{3}\left(p_{4}-\theta\right)\right)}
$$

where $\mathrm{T}(\theta)$ is the resistance torque $(\mathrm{N} \mathrm{m})$ of the shoulder complex joint robot. The role of parameters $\mathrm{p} 1$ and $\mathrm{p} 3$ is to determine the degree of resistance torque at the end point of the joint angle, whereas parameters $\mathrm{p} 2$ and $\mathrm{p} 4$ designate the functional joint angle range. Figure 3 represents a graph of the reproduction of resistance torque with respect to varying joint angles. Change in $\mathrm{p} 1$ and p3 leads to change in the graph slope. Parameter p2 and $\mathrm{p} 4$ are the coefficients indicating the starting point of the joint extension and the end point of the joint flexion. Based on the opinions of the experts (caregivers) who participated in this experiment, these parameters were determined to allow the robot imitate an elderly person and to reproduce the relevant joint movement range with limited motion.

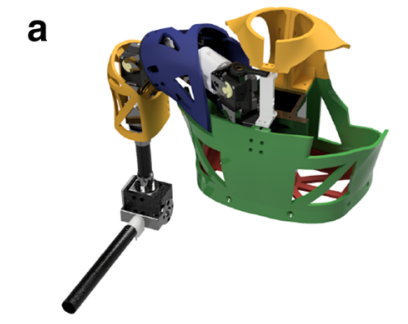

Front view

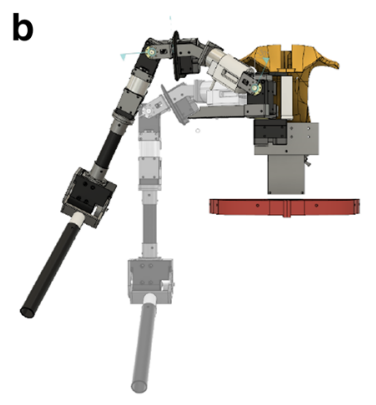

Elevation and depression (ED)
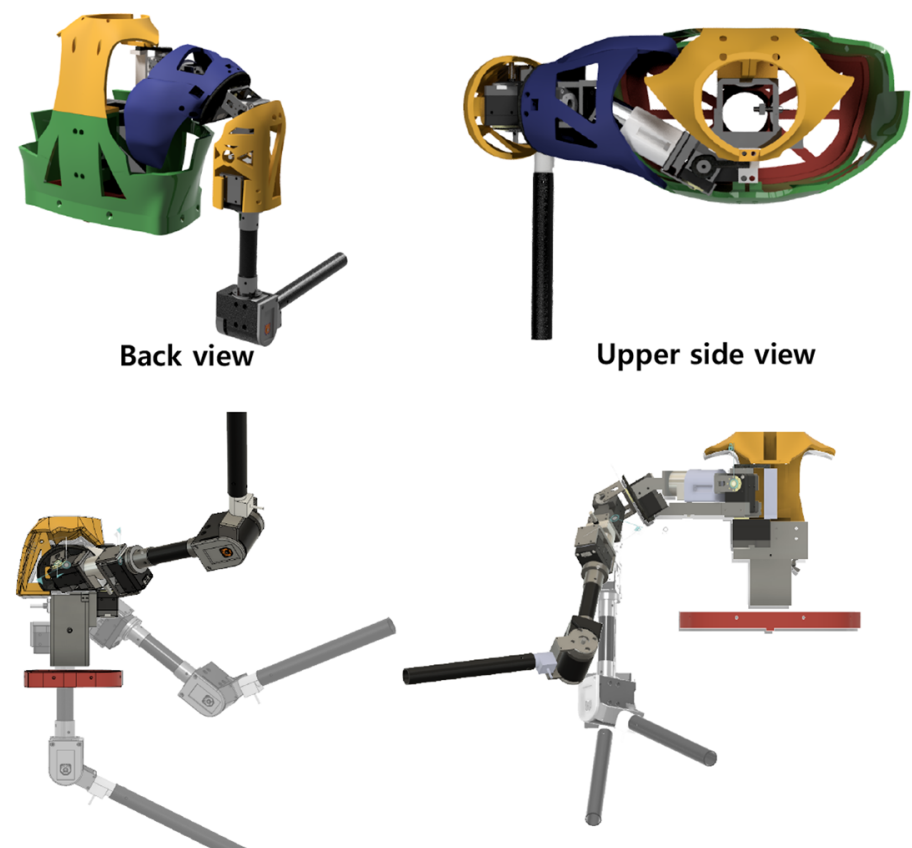

Extension and flex (EF)
Lateral and medial rotation (LM)

Fig. 2 Upper limb of the care training assistant robot. a 3D modeling views. $\mathbf{b}$ Mechanisms of the upper limb of care training assistant robot 


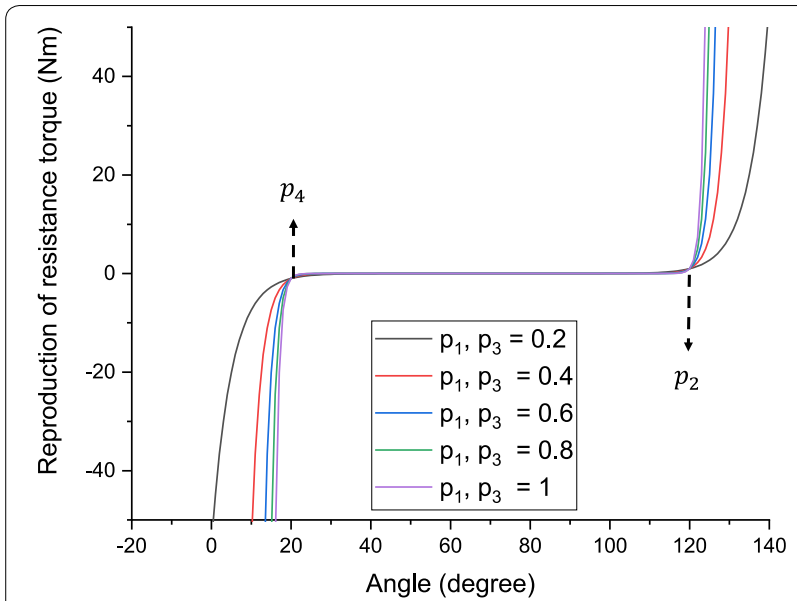

Fig. 3 Example of resistance torque graph of joint resistance torque with respect to joint angle
Table 1 A detailed description of the expert experience

\begin{tabular}{|c|c|c|}
\hline Expert & Experience & Gender \\
\hline Expert 1 (EXP 1) & Experience in nursing care and orthopedics & Male \\
\hline Expert 2 (EXP 2) & $\begin{array}{l}\text { Experience in ROM exercise } \\
\text { Working as physical therapist in a hospital }\end{array}$ & Female \\
\hline Expert 3 (EXP 3) & $\begin{array}{l}\text { Has a national therapist certification } \\
\text { Has } 2 \text { years of experience in ROM training }\end{array}$ & Female \\
\hline Expert 4 (EXP 4) & $\begin{array}{l}\text { Working as a speech therapist } \\
\text { Has experience in ROM training of the } \\
\text { shoulders and fingers }\end{array}$ & Female \\
\hline Expert 5 (EXP 5) & Working as a nursing care specialist & Female \\
\hline
\end{tabular}

a consent form, while researchers tried to ensure their safety. Furthermore, the entire experimental procedure was approved by the institutional review board (IRB) of the Ritsumeikan University (BKC-2018-059).

\section{Procedures}

All experts performed feasibility experiments consisting of three ROM exercises-elevation and depression (ED), extension and flexion (EF), and lateral and medial rotations (LM) - as shown in Fig. 4. These ROM exercises are frequently performed for the rehabilitation of people suffering from shoulder diseases. In the ED exercise, elevation and depression represent the upward and downward movement of body structures, respectively; for example, the depression of a sterno-clavicular joint corresponds to the arm moving vertically downward. The EF exercise constitutes increasing and decreasing the angle between the two bones connected by the glenohumeral joint [28]. The LM exercise refers to the lateral and medial arm rotations of the humeral around its longitudinal axis. The ROM exercises were performed five times each, in the order of ED, EF, and LM, and in certain cases, were performed with the robot lying on the bed, based on certain instructions $[29,30]$. All movements started at the point where the pre-defined joint angle was $0^{\circ}$.

Did experts conduct ROM exercise using the robot objectively when performing the care education? Do the quantitative data obtained from the experts vary?

ii. Could the experts be satisfied with feasibility and possibility of the proposed robot through this pilot experiment?

\section{Participants}

Five experts (caregiver and/or therapist) with up to 3 years of experiences in rehabilitation center and hospital, were participated in this experiment, as shown in Table 1, all of whom agreed to participate by signing

\section{Evaluation method}

For evaluating the care skill, each of the five experts completed the care education with three types of ROM exercise using the elderly simulated robot. To analyze the relationship of the results and significance effect among experts, we used the statistical method of two-way repeated measurement analysis of variance (ANOVA) test, where, statistical analyses were performed using MATLAB $^{\circledR} 2019$ software. The P values (probability) were considered statistically significant when: ${ }^{*} \mathrm{P}<0.05$ and ${ }^{* *} \mathrm{P}<0.001$. All data were expressed as mean \pm standard deviation. 
a

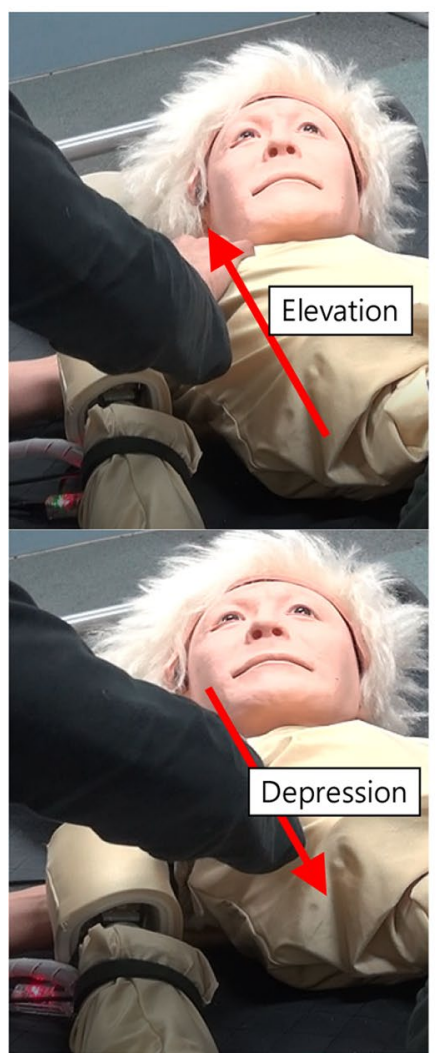

b

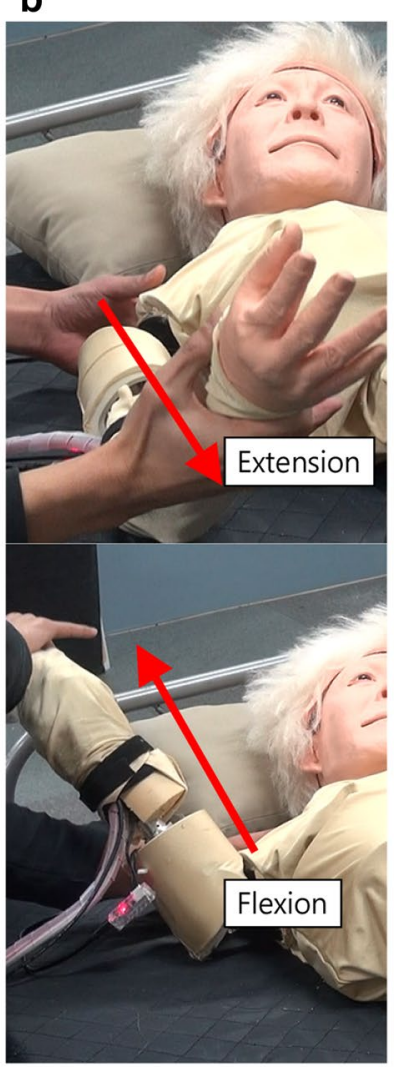

c

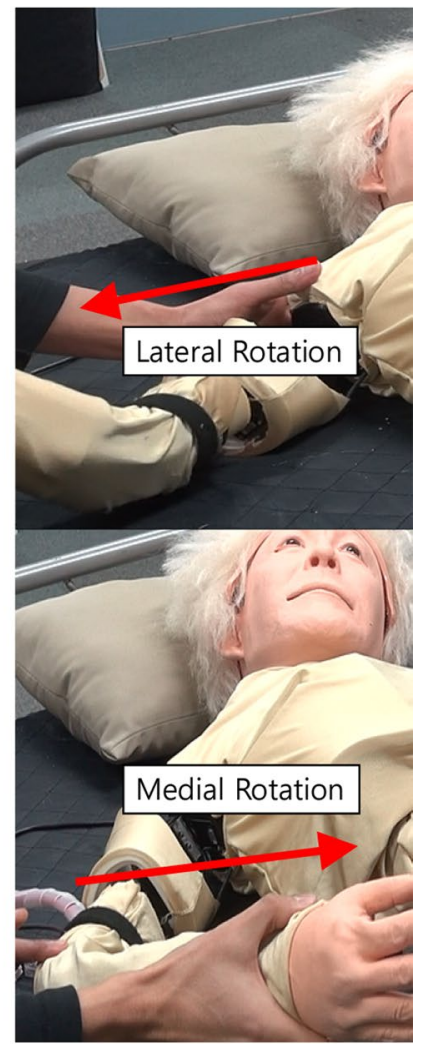

Fig. 4 Typical example of the motion range (ROM) exercise. a Elevation-depression; b extension-flexion; c lateral rotation-medial rotation

\section{Results and discussion}

In this study, based on the obtained data, the experiment was conducted to evaluate the necessity and the feasibility of the proposed care training robot for the care education. Figures 5, 6, and 7 demonstrate the typical examples of the obtained raw data of angle and torque from experts in ED, EF, and LM, respectively.

Figure 5 shows the typical example of ED exercise from the experts (EXP 1, EXP2, and EXP 3), which represented the angle values of $5 \mathrm{DOF}(\mathrm{SC} 1, \mathrm{SC} 2, \mathrm{GH} 1$, $\mathrm{GH} 2, \mathrm{GH} 3$ ), and the torque values of $\mathrm{SC}$ and $\mathrm{GH}$ joints. The obtained data from EXP 4 and EXP 5 were excluded due to high noise. In ED exercise, the joint movements of $\mathrm{SC} 1$ and GH2 were clearly represented, in which, the experts performed each cycle of ED exercise in constant motion range. However, the experts differed slightly in the motion range of the joints.

The experts performed the EF exercise based on the GH1 and SC1 joints. As shown in Fig. 6, the movements performed by EXP1, EXP3 and EXP5 were almost the same as each other. When EXP 2 performed the exercise, however, the joint angles were not constant in the time domain, because EXP 2 did not perform a complete movement from flexion to extension, i.e., a contraction was performed again after a slight joint relaxation.

In the LM exercise (Fig. 7), the results of all experts were significantly different. The EXP1 and EXP5 had a constant joint angle amplitude and period for each motion cycle. The period values of EXP 1 and EXP 5 were faster than that of the EXP 2 and EXP 3, while the EXP2 and EXP3 were observed to had a longer period of joint rotation from lateral to medial direction.

Figure 8 shows the example of angle curves between $\mathrm{SC} 1$ and $\mathrm{GH} 2$ during the ED exercise performance by experts. Typically, the experts hold the robot shoulder (grip position 1: G1) with their right hand, and the arm (G2) with their left hand when performing the ED exercise as shown in Fig. 8a. Figure 8b presents the mechanism of the ED exercise, while the joints of $\mathrm{SC} 1$ and $\mathrm{GH} 2$ were involved when performing ED movement with robot based on G1 and G2. In the depression movement, the expert lowers the SC1 joint of the robot while lowering the GH2 joint simultaneously, and vice versa. Figure $9 \mathrm{c}-\mathrm{e}$ shows the angle curves of $\mathrm{SC} 1$ and $\mathrm{GH} 2$ joints. In all three tests (T1, T2, and T3), EXP 1 (Fig. 8c) and EXP 3 (Fig. 8e) had a constant angle amplitude and 


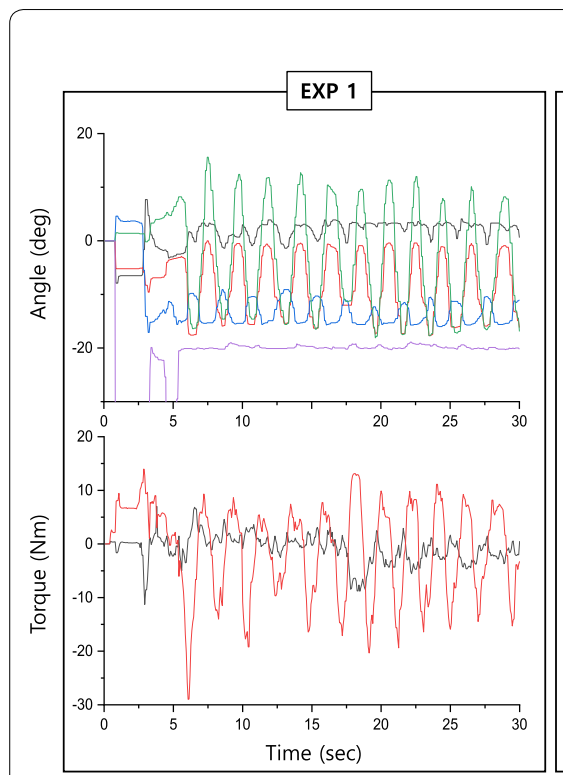

\section{Elevation and depression (ED)}
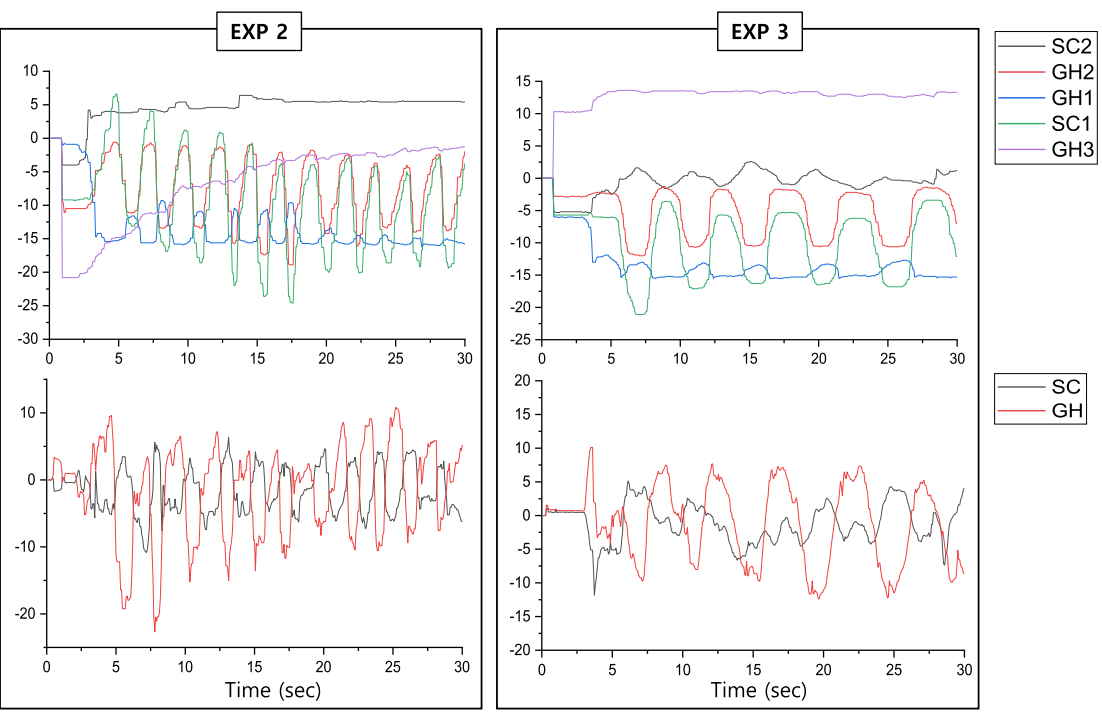

Fig. 5 Experiment values of the raw data of angle and torque in elevation and depression (ED) exercise from the experts (EXP expert, SC sterno-clavicular joint, GH glenohumeral joint). The $x$-axis indicates the time (unit: second). The top and bottom y-axes represent the angle (unit: degree) and torque (unit: $\mathrm{Nm}$ ), respectively

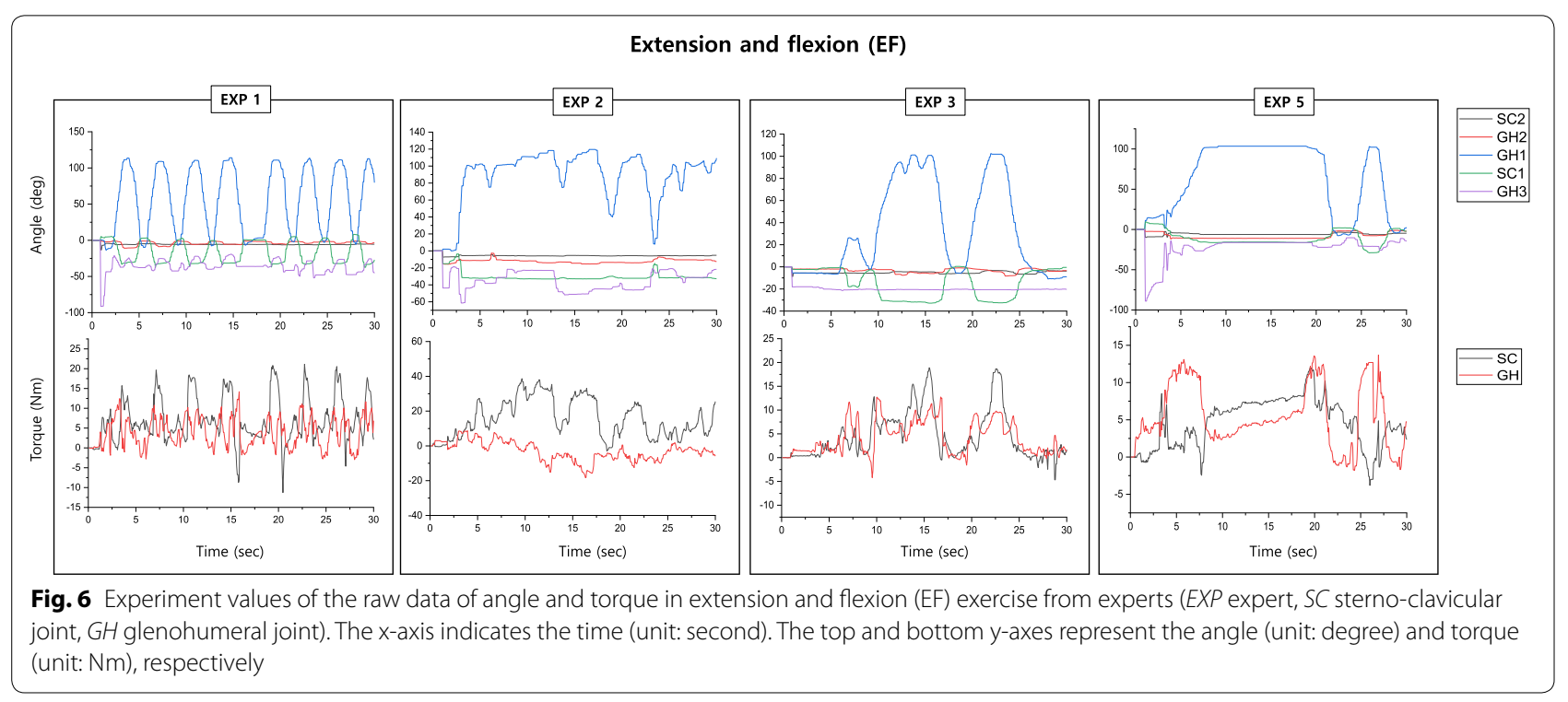

movement period for each test. However, the EXP 2 showed that the T1 movement was in the opposite direction of T2 and T3 (Fig. 8d). EXP 2 lowered the shoulder joint (GH2) when raising the clavicle ( $\mathrm{SC} 1)$ during the first test (T1), and raised it when lowering the clavicle joint.

Figure 9 shows the examples of angle curves between $\mathrm{SC} 1$ and GH1 during the EF exercise performance by the experts. When performing the EF exercise, the experts generally tend to hold the robot shoulders (G1) with their left hand, and the arm (G2) with their right hand, as shown in Fig. 9a. Then, the joints of SC1 and GH1 were involved when performing EF movement with G1 and G2, as shown in Fig. 9b. In the flexion movement, the $\mathrm{GH} 1$ and the $\mathrm{SC} 1$ joints were raised simultaneously, and vice versa. Figure $9 \mathrm{c}-\mathrm{e}$ shows the angle curves of $\mathrm{SC} 1$ and GH1 joints. EXP 1, EXP 2, and EXP 3 had the constant amplitude of angle range and the movement period 

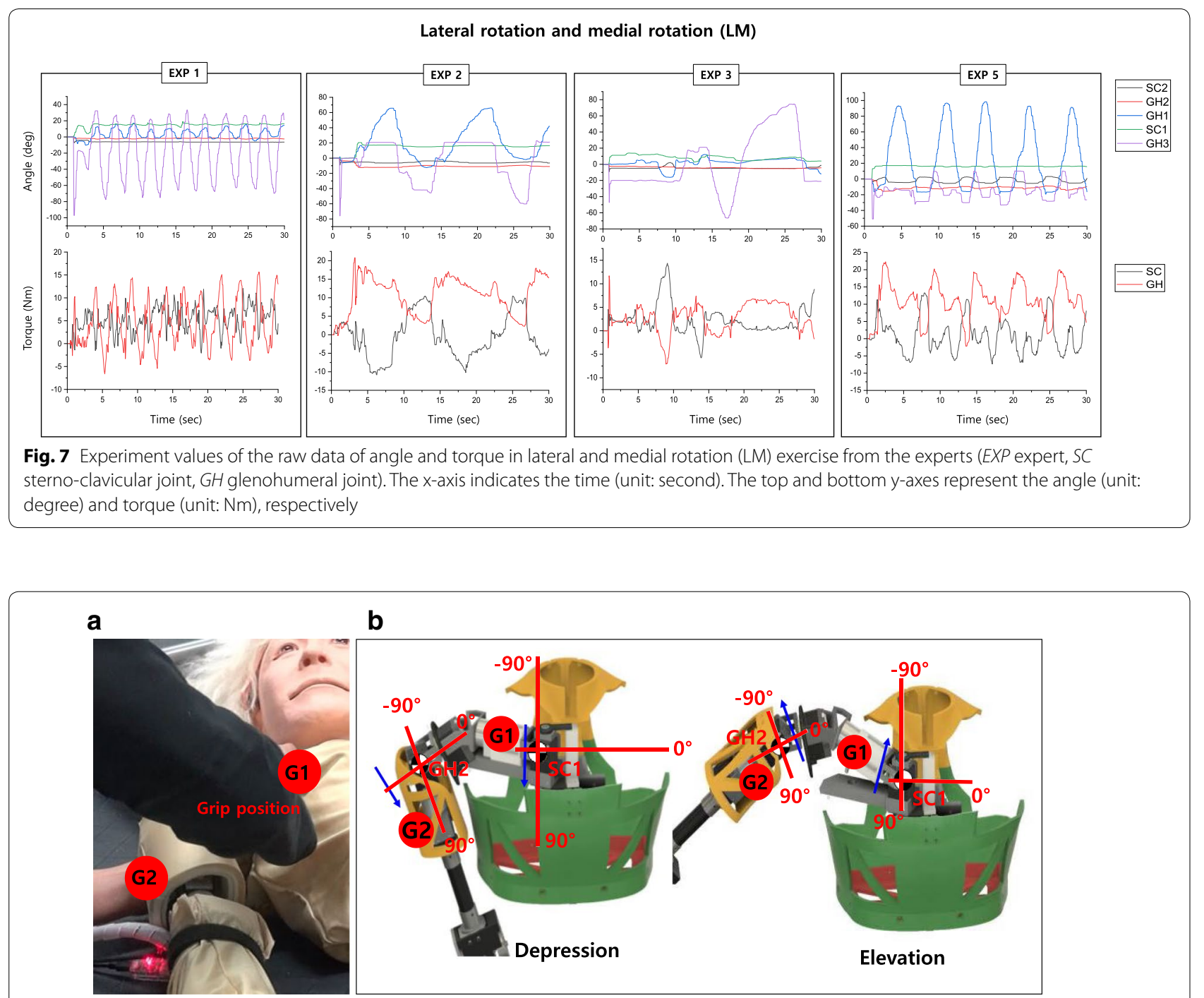

\section{b}

C

EXP 1

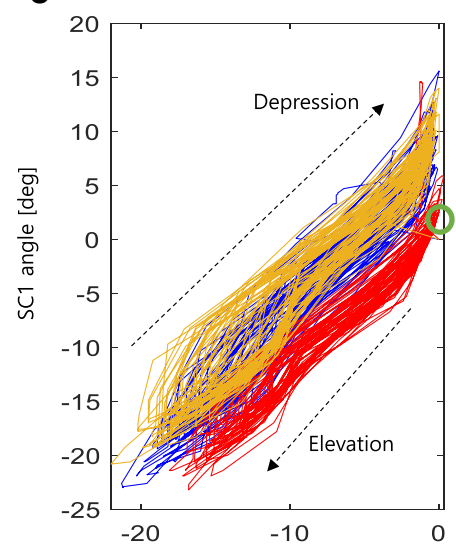

$\mathrm{GH} 2$ angle [deg]

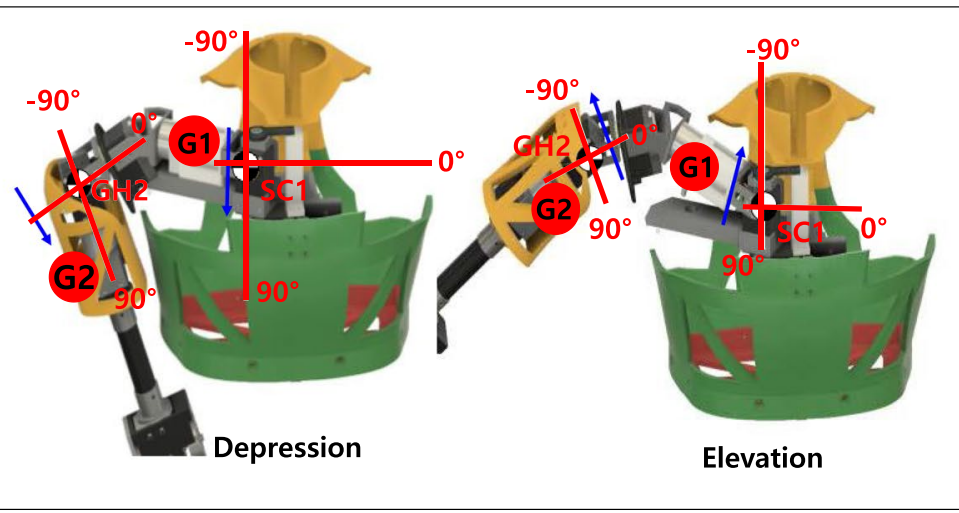

d
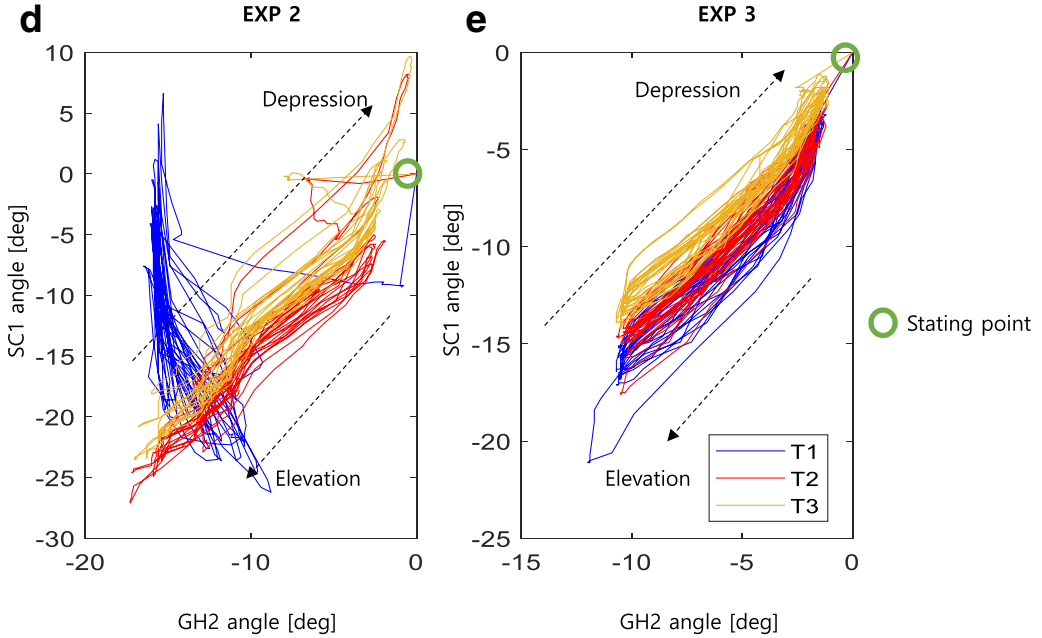

Fig. 8 Example of sterno-clavicular and glenohumeral joints in elevation and depression (ED) exercise from the experts: a explanation of grip positions; $\mathbf{b}$ mechanism of elevation and depression; $\mathbf{c}$ angle curves between SC1 and GH2 joints. EXP expert, SC sterno-clavicular joint, GH glenohumeral joint, $T n$ the number of tests. The green circle indicates the starting point of the exercise 
a

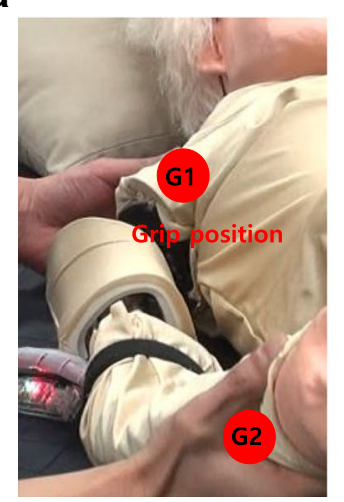

b

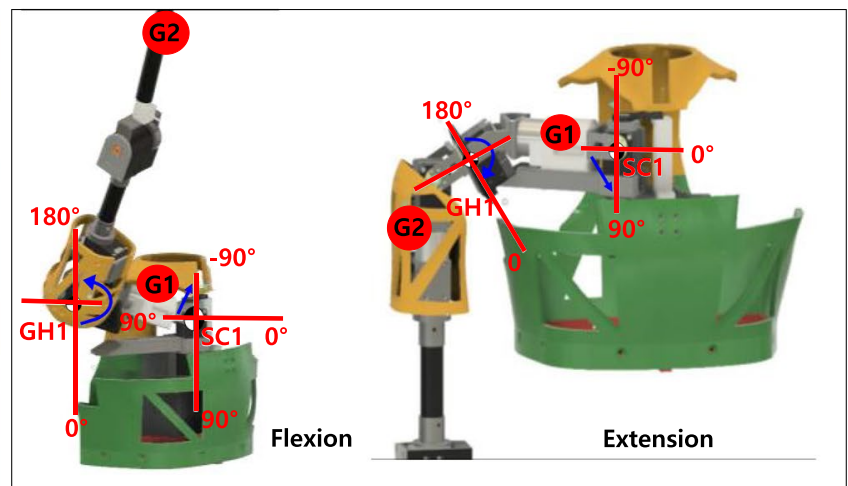

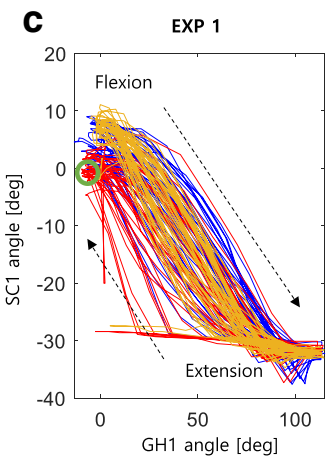
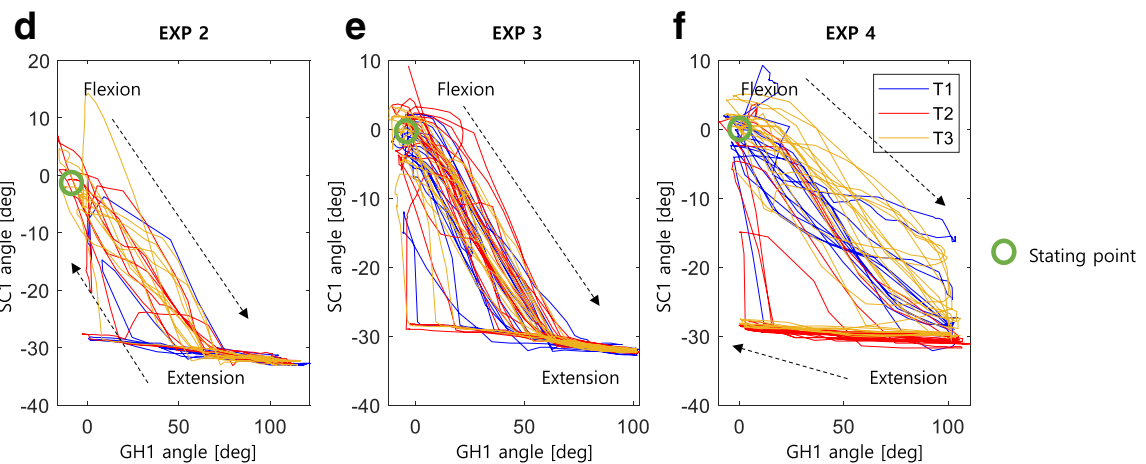

Fig. 9 Example of sterno-clavicular and glenohumeral joints in extension and flexion (EF) exercise from the experts: a explanation of grip positions; b mechanism of elevation and depression; c angle curves between $\mathrm{SC} 1$ and $\mathrm{GH} 2$ joints. EXP expert, SC sterno-clavicular joint, GH glenohumeral joint, Tn the number of tests. The green circle indicates the starting point of the exercise

for each test. In the result of EXP 4, the subject did not bend the robot joints as much as possible when the angle changed from extension to bend, and moved only about -10 to 10 degrees, thus, exhibiting a difference from the results of other experts.

Figure 10 shows an example of expert data in an LM exercise. In the LM movement, experts typically hold the robot shoulders (G1) with left hand and the robot arm (G2) with right hand, as shown in Fig. 10a. The joints of G1 and GH2 were involved when performing LM motion while holding G1 and G2 as shown in Fig. 10b. In medial rotation, the $\mathrm{GH} 2$ joints were rotated inward and the GH1 joints were lowered, as the GH1 joint was raised while rotating the $\mathrm{GH} 2$ joint outward in lateral rotation. The results of the movement angle based on the joints of GH1 and GH2 are shown in Fig. 10c-f. In all three testing, although each expert showed a certain amplitude and period for each test, the angle pattern of all experts demonstrated a different movement.

Figure 11 shows the results of the statistical analyses of ED, EF, and LM. To statistically evaluate the acquired data, we extracted all the eleven parameters: maximum angle of SC1 (MAX_SC1), maximum angle of SC2 (MAX_SC2), maximum angle of GH1 (MAX GH1), maximum angle of GH2 (MAX_GH2), maximum angle of GH3 (MAX_GH3), minimum angle of SC1 (MIN_SC1), minimum angle of SC2 (MIN_SC2), minimum angle of GH1 (MIN_GH1), minimum angle of GH2 (MIN_GH2), minimum angle of GH3 (MIN GH3), and the interval between consecutive cycles (INTV). Based on these parameters, an ANOVA test was conducted to analyze statistically significant differences between the experts. It was observed that all parameters except INTV $(\mathrm{P}=0.138292)$ in the EF exercise exhibited significant differences between the experts.

The findings of our experimental study suggest that although experts have several years of experiences, they may perform care and treatment differently from each other. As different methods of care and treatment may introduce a detrimental effect on the joints and muscles of an elderly person, a customized and suitable careeducation method for individuals should be provided on the basis of quantitative data analysis. 

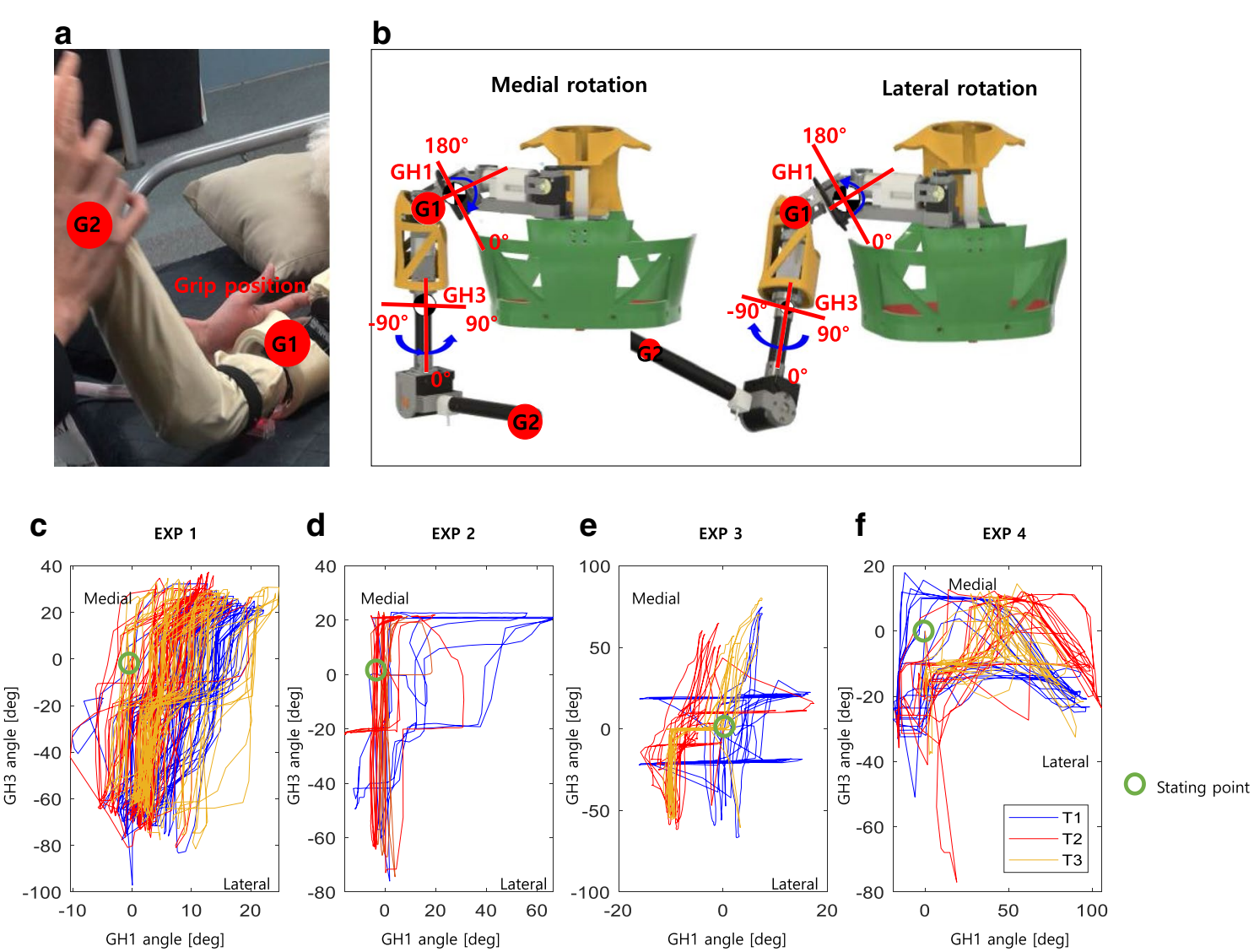

Fig. 10 Example of glenohumeral joints in lateral and medial rotation (LD) exercise from the experts: a explanation of grip positions; $\mathbf{b}$ mechanism of elevation and depression; c angle curves between SC1 and GH2 joints. EXP expert, GH glenohumeral joint, Tn the number of tests. The green circle indicates the starting point of the exercise

\section{Conclusion and future work}

The study on development of simulated robot makes an important contribution to improve care skills of caregivers more accurately in the care education. This research aimed to experimentally investigate whether there was a difference in the quantitative data results among the experts during the care training.

Firstly, we developed the care training assistant robot with multi-DOF at the shoulder complex joint in the care education and training for caregivers. To investigate the necessity and the feasibility of care training using the proposed method, related experiments were conducted with five elderly care experts, who performed three types of ROM exercises using the proposed system. Our findings suggested that even experts with many years of experiences may have different methods, thus, introducing a negative impact on care and treatment. Therefore, the necessity and the feasibility of the care training assistant robot should be utilized for the improvement of accurate and elaborated care skills in the elderly care education.

By considering the broader application scope of the proposed system, however, it is evident that the experimental subjects were not suitable for drawing generalized conclusions. Therefore, additional test subjects will need to be recruited to perform more experiments, while the investigation of specific medical symptoms (i.e., stiffness, contracture, and muscle weakness) is necessary for various elderly subjects. Furthermore, future research should focus on investigating whether the usage of the proposed system by the experts leads to improvements to and generalization of the care and rehabilitation skills of caregivers. Comparisons should also be made between the results obtained from actual elderly patients and the proposed robot patient. Finally, it is necessary to perform various ROM exercises and care activities involving different postures (e.g. sitting, standing, etc.) because the ROM exercises were conducted for a single case (lying in bed) in this study. 
a

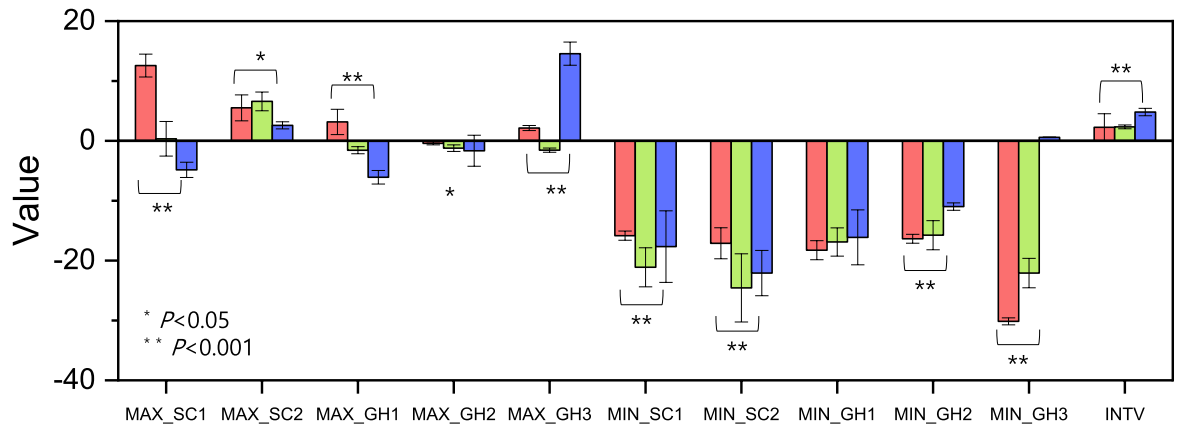

$\square$ Expert 1

Expert 2

Expert 3

b

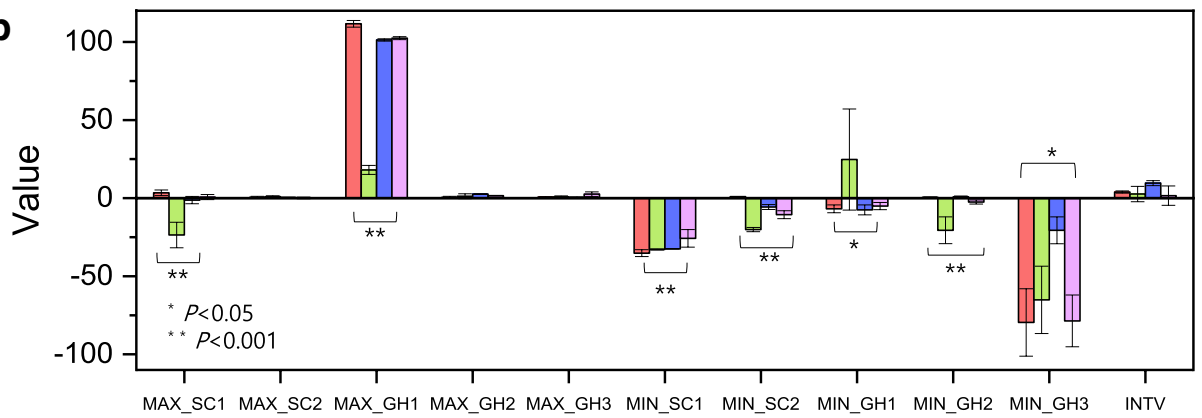

$\square$ Expert 1

$\square$ Expert 2

$\square$ Expert 3

$\square$ Expert 5

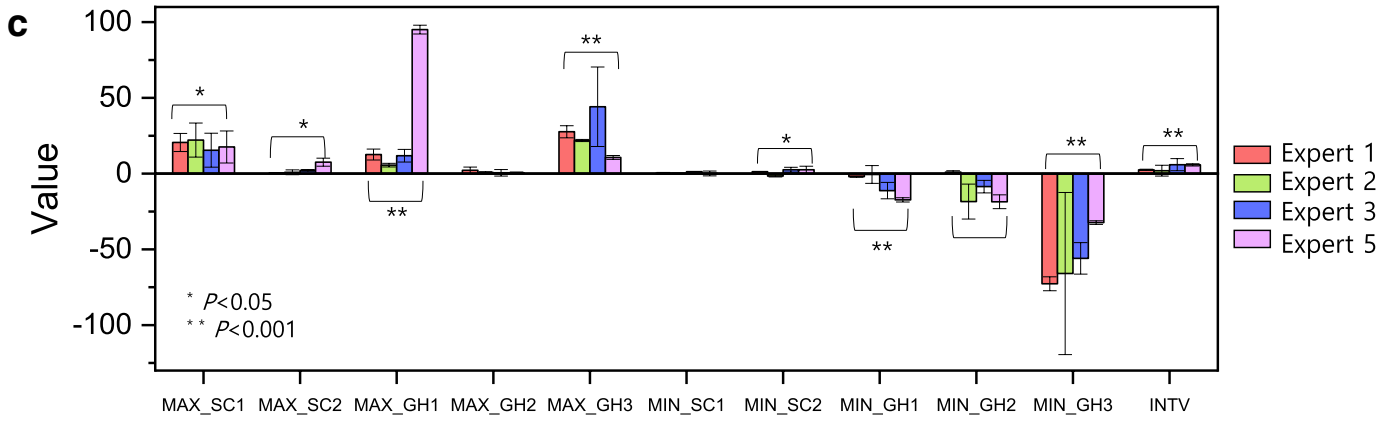

Parameters

Fig. 11 Results of statistical analysis with respect to all 11 parameters, a elevation and depression (ED); b extension and flexion (EF); c lateral- and medial- rotation (LM) exercise from the experts. The MAX and MIN with underscore represent the maximum and minimum joint angles, while INTV indicates the interval of each cycle

\section{Acknowledgements}

The authors would like to gratefully acknowledge the financial support of JSPS KAKENHI (Grant Number JP17K00372) and R-GIRO.

\section{Authors' contributions}

Authors, including ML, KA, HY, and JL, contributed to the design of the work, and $M L$ and $K A$ contributed to the acquisition, analysis, and interpretation of the data for the work. ML, HY, and JL contributed to writing and revising the work for important intellectual content. All authors read and approved the final manuscript.

\section{Funding}

This work was supported by JSPS KAKENHI (Grant Number JP17K00372) and R-GIRO.

\section{Availability of data and materials}

Not applicable.
Ethics approval and consent to participate

Ethical approval was granted by the Ethics Committee of Ritsumeikan University.

\section{Consent for publication}

Written informed consent for the publication of experimental details was obtained from all subjects.

\section{Competing interests}

The authors declare that they have no competing interests.

\section{Author details}

${ }^{1}$ Graduate School of Information Science and Engineering, Ritsumeikan University, Kusatsu-Shi, Shiga-Ken 5258577, Japan. ${ }^{2}$ College of Information Science and Engineering, Ritsumeikan University, Kusatsu-Shi, Shiga-Ken 5258577, Japan. 
Received: 30 September 2019 Accepted: 20 February 2020

Published online: 03 March 2020

\section{References}

1. Matsumoto Y, Ogata K, Kajitani I, Homma K, Wakita Y (2018) Evaluating robotic devices of non-wearable transferring aids using whole-body robotic simulator of the elderly. In: 2018 IEEE/RSJ International Conference on Intelligent Robots and Systems (IROS), pp 6174-6179

2. Aung T, Montagu D, Schlein K, Khine TM, McFarland W (2012) Validation of a new method for testing provider clinical quality in rural settings in low- and middle-income countries: the observed simulated patient. PLoS ONE 7(1):e30196

3. Yaeger KA et al (2004) High-fidelity simulation-based training in neonatal nursing. Adv Neonatal Care 4(6):326-331

4. Cooper JB, Taqueti VR (2008) A brief history of the development of mannequin simulators for clinical education and training. Postgrad Med J 84(997):563-570

5. Johnsson ACE, Kjellberg A, Lagerström MI (2006) Evaluation of nursing students' work technique after proficiency training in patient transfer methods during undergraduate education. Nurse Educ Today 26(4):322-331

6. Huang $Z$ et al (2015) Design and evaluation of robot patient for nursing skill training in patient transfer. Adv Robot 29(19):1269-1285

7. Lee M, Yamazoe H, Lee JH (2019) Elderly care training using real-time monitoring system with care training assistant elbow robot (CaTARo-E). In: 2019 16th International Conference on Ubiquitous Robots (UR), pp 259-264

8. Fujisawa T et al (2007) Basic research on the upper limb patient simulator. In: 2007 IEEE 10th international conference on rehabilitation robotics, pp 48-51

9. Mouri T, Kawasaki H, Nishimoto Y, Aoki T, Ishigure Y (2007) Development of robot hand for therapist education/training on rehabilitation. In: 2007 IEEE/RSJ international conference on intelligent robots and systems, pp 2295-2300

10. Wang C et al (2013) Development of a human-like neurologic model to simulate the influences of diseases for neurologic examination training. In: 2013 IEEE international conference on robotics and automation, pp 4826-4831

11. Huang Z et al (2014) Robot patient for nursing self-training in transferring patient from bed to wheel chair. Springer, Cham, pp 361-368

12. Kitagawa Y, Ishikura T, Song W, Mae Y, Minami M, Tanaka K (2009) Humanlike patient robot with chaotic emotion for injection training. In: 2009 ICCAS-SICE, pp 4635-4640

13. Takashina T, Shimizu M, Katayama H (1997) A new cardiology patient simulator. Cardiology 88(5):408-413

14. Gordon MS (1974) Cardiology patient simulator: development of an animated manikin to teach cardiovascular disease. Am J Cardiol 34(3):350-355

15. Wang $C$ et al (2012) Development of an arm robot for neurologic examination training. In: 2012 IEEE/RSJ international conference on intelligent robots and systems, pp 1090-1095
16. Takanobu $\mathrm{H}$ et al (2007) Dental patient robot as a mechanical human simulator. In: 2007 IEEE international conference on mechatronics, pp 1-6

17. Gerling GJ, Rigsbee S, Childress RM, Martin ML (2009) The design and evaluation of a computerized and physical simulator for training clinical prostate exams. IEEE Trans Syst Man Cybern A Syst Hum 39(2):388-403

18. Ren Y, Kang SH, Park HS, Wu YN, Zhang LQ (2013) Developing a multijoint upper limb exoskeleton robot for diagnosis, therapy, and outcome evaluation in neurorehabilitation. IEEE Trans Neural Syst Rehabil Eng 21(3):490-499

19. Lin C et al (2019) Effect of practice on similar and dissimilar skills in patient transfer through training with a robot patient. Adv Robot 33(6):278-292

20. Kim J, Park HS, Damiano DL (2011) Accuracy and reliability of haptic spasticity assessment using HESS (Haptic Elbow Spasticity Simulator). In: 2011 annual international conference of the IEEE engineering in medicine and biology society, pp 8527-8530

21. Huang $Z$ et al (2017) Impact of using a robot patient for nursing skill training in patient transfer. IEEE Trans Learn Technol 10(3):355-366

22. Park HS, Kim J, Damiano DL (2012) Development of a haptic elbow spasticity simulator (HESS) for improving accuracy and reliability of clinical assessment of spasticity. IEEE Trans Neural Syst Rehabil Eng 20(3):361-370

23. Noh Y et al (2008) WKA-1R robot assisted quantitative assessment of airway management. Int J Comput Assist Radiol Surg 3(6):543-550

24. Murata K, Yamazoe H, Chung MG, Lee JH (2018) Elderly care training robot for quantitative evaluation of care operation-Development of robotic elbow joint to imitate elderly people's elbow. In: 2017 IEEE/ SICE international symposium on system integration (SII), vol 2018, pp 1028-1033

25. Lee M, Murata K, Ameyama K, Yamazoe H, Lee JH (2019) Development and quantitative assessment of an elbow joint robot for elderly care training. Intell Serv Robot 12:1-11

26. Human characteristics database | consumer product safety | National Institute of Technology and Evaluation (NITE).https://www.nite.go.jp/en/ jiko/s_standard/human_db/index.html. Accessed 06 May2019

27. Yamazaki N, Tanaka T (2006) Development of a human joint imitated dummy. Biomechanisms 18:175-185

28. Werner BC, Kuenze CM, Griffin JW, Lyons ML, Hart JM, Brockmeier SF (2013) Shoulder range of motion. Orthop J Sport Med 1(4_suppl):232596711350010

29. BS Medicine. Shoulder range of motion exercises. http://www.bosto nsportsmedicine.com/rehabilitation-protocols/shoulder-stretching-exerc ises/

30. Passive shoulder range of motion-St. Jude Children's Research Hospital. https://www.stjude.org/treatment/patient-resources/caregiver-resou rces/patient-family-education-sheets/rehabilitation/passive-shoulderrange-of-motion.html. Accessed 242019

\section{Publisher's Note}

Springer Nature remains neutral with regard to jurisdictional claims in published maps and institutional affiliations.

\section{Submit your manuscript to a SpringerOpen ${ }^{\odot}$ journal and benefit from:}

- Convenient online submission

- Rigorous peer review

- Open access: articles freely available online

- High visibility within the field

- Retaining the copyright to your article

Submit your next manuscript at springeropen.com 